\title{
Phase-Imprinting of Bose-Einstein Condensates with Rydberg Impurities
}

\author{
Rick Mukherjee, ${ }^{1}$ Cenap Ates, ${ }^{2}$ Weibin $\mathrm{Li}^{2},{ }^{2}$ and Sebastian Wüster ${ }^{1}$ \\ ${ }^{1}$ Max Planck Institute for the Physics of Complex Systems, Nöthnitzer Strasse 38, 01187 Dresden, Germany \\ ${ }^{2}$ School of Physics and Astronomy, University of Nottingham, Nottingham NG7 2RD, United Kingdom
}

(Received 16 February 2015; published 21 July 2015)

\begin{abstract}
We show how the phase profile of Bose-Einstein condensates can be engineered through its interaction with localized Rydberg excitations. The interaction is made controllable and long range by off-resonantly coupling the condensate to another Rydberg state with laser light. Our technique allows the mapping of entanglement generated in systems of few strongly interacting Rydberg atoms onto much larger atom clouds in hybrid setups. As an example we discuss the creation of a spatial mesoscopic superposition state from a bright soliton. Additionally, the phase imprinted onto the condensate using the Rydberg excitations is a diagnostic tool for the latter. For example, a condensate time-of-flight image would permit reconstructing the pattern of an embedded Rydberg crystal.
\end{abstract}

DOI: 10.1103/PhysRevLett.115.040401

PACS numbers: 03.75.Lm, 32.80.Ee, 32.80.Qk

Introduction.-The imprinting of tailored phase profiles onto the complex order parameter of a Bose-Einstein condensate (BEC) [1] is a versatile tool for the creation of topological states such as solitons [2,3] and vortices [1], or even Skyrmions [4]. More generally, imprinting allows the transfer of the BEC into a desired state of atom flow or motion. Typically, the phase is generated purely optically using lasers. We propose engineering phases exploiting the interactions between BEC atoms that are Rydberg dressed [5-13] and resonantly excited Rydberg atoms [14-18]. This phase-imprinting will rely on the matter-wave coherence of the condensate and thus represent an instance of genuine Rydberg BEC physics.

We show that phase-imprinting creates a versatile interface between ultracold Rydberg and BEC physics. First, it allows entangled Rydberg states [19-21] to be mapped onto the many-body wave function of the condensate. As one example, we discuss how to turn an atomic Bell state $|+\rangle=(|R g\rangle+|g R\rangle) / \sqrt{2}$ (with two atomic electronic states $|g\rangle,|R\rangle$ ) into a spatial mesoscopic superposition state in the position of a single BEC bright soliton, akin to the proposal of Ref. [22]. Second, phase-imprinting represents a tool to probe Rydberg electronic states via their effect on a condensate [18,23-27]. To demonstrate, we show signatures of Rydberg crystals [28] in the expected condensate time of flight spectra.

Our scheme relies on weakly admixing Rydberg character to all of the atoms in a condensate cloud through far offresonant laser coupling between their stable ground state and a highly excited Rydberg state [6,7], as experimentally demonstrated for two atoms [13]. All of these "dressed" atoms then interact with some previously prepared, fully excited atoms in a different Rydberg state, referred to as impurity or control atoms depending on whether they are inside or outside the condensate. The atomic species of impurities may be identical to condensate atoms.
The interaction involving impurities can be made dominant over the simultaneously induced long-range condensate self-interaction [6,7]. Using this we demonstrate the imprinting of sizable phases for realistic parameters.

The condensate phase is also affected by direct collisions of the Rydberg electron with condensate atoms in the

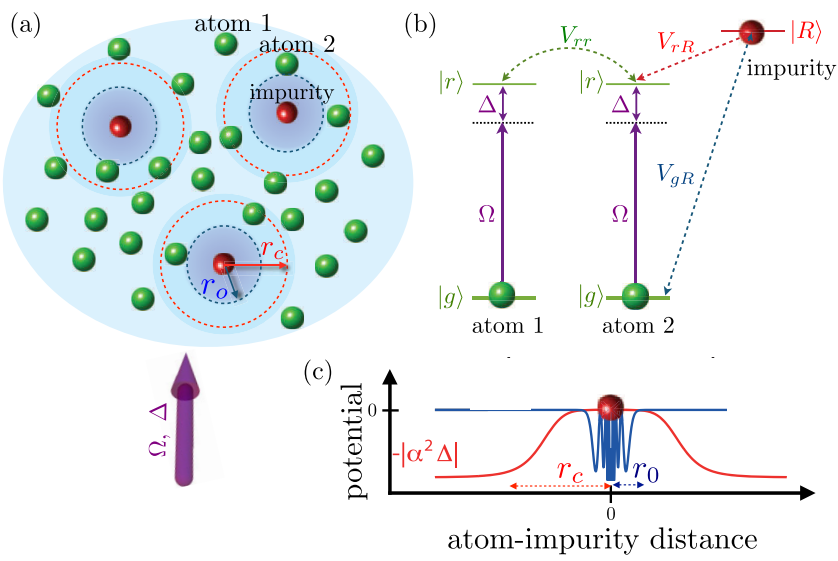

FIG. 1 (color online). (a) The setup. Several Rydberg excited impurities (the red balls) are embedded in a Bose-Einstein condensate. The condensed atoms (the green balls) mainly interact with impurities through dressing induced long-range interactions of characteristic range $r_{c}$ (the red dashed circles). At distances $d$ within the Rydberg orbit, $d<r_{0}$, collisions with Rydberg electrons are also relevant (the blue dashed circles). After an imprinting period, the condensate phase (blue shades) will be modified only in the vicinity of impurities (dark blue). (b) Level scheme for three representative atoms. Interactions $V_{r r}$ give rise to long-range interactions between dressed condensate atoms, as in Refs. [6,7]. Interactions $V_{r R}$ and $V_{g R}$ give rise to stronger potentials between dressed condensate atoms and impurities, which are the main focus here. (c) Sketch of these potentials $U_{\text {eff }}^{(2)}$ (red, see text) and $V_{g R}$ (blue) near one of the impurities. $\alpha=\Omega /(2 \Delta)$ quantifies the degree of the Rydberg admixture. 
ground state, without dressing coupling. This can be exploited to visualize the electron orbital through condensate densities $[18,27]$. In contrast, the imprinting through dressed interactions discussed here extends the spatial scale, smoothness, and controllability of phase profiles. The use of Rydberg impurities and effectively one-(1D) or two-dimensional (2D) condensates in this Letter circumvents some of the interaction strength and many-body related problems of dressing discussed in Ref. [12].

Interactions between Rydberg impurities and dressed atoms.-Consider a gas of $N \mathrm{Rb}$ atoms with mass $M$ at locations $\mathbf{R}_{n}$, as depicted in Fig. 1(a). They may be in any of three electronic states: ground state $|g\rangle$, Rydberg state $|r\rangle=|\nu s\rangle$, or Rydberg state $|R\rangle=\left|\nu^{\prime} s\right\rangle$, with the principal quantum numbers $\nu, \nu^{\prime}$, where $\nu<\nu^{\prime}$, as shown in Fig. 1(b). The label $s$ implies the angular momentum $l=0$. We write the system Hamiltonian $\hat{H}_{\text {tot }}=\hat{H}_{0}+\hat{H}_{\text {dress }}+\hat{H}_{\text {int }}$, using the notation $\hat{\sigma}_{k k^{\prime}}^{(n)}=|k\rangle\left\langle k^{\prime}\right|$, where $k, k^{\prime} \in\{g, r, R\}$ and $\hat{\sigma}_{k k^{\prime}}^{(n)}$ acts on atom $n$ only:

$$
\begin{aligned}
\hat{H}_{0} & =\sum_{n=1}^{N}\left[-\frac{\hbar^{2} \nabla_{\mathbf{R}_{n}}^{2}}{(2 M)}+W\left(\mathbf{R}_{n}\right) \hat{\sigma}_{g g}^{(n)}\right], \\
\hat{H}_{\text {dress }} & =\sum_{n=1}^{N}\left[\frac{\Omega(t) \hat{\sigma}_{r g}^{(n)}}{2}+\text { H.c. }-\Delta \hat{\sigma}_{r r}^{(n)}\right], \\
\hat{H}_{\mathrm{int}} & =\sum_{a, b \in\{g, r, R\}} \sum_{n \neq m=1}^{N} V_{a b}\left(d_{n m}\right) \hat{\sigma}_{a a}^{(n)} \hat{\sigma}_{b b}^{(m)} .
\end{aligned}
$$

Ground state atoms experience an external trapping potential $W\left(\mathbf{R}_{n}\right)$. The state $|g\rangle$ is coherently coupled to $|r\rangle$ with Rabi frequency $\Omega(t)$ and detuning $\Delta$. Defining $d_{n m}=\left|\mathbf{R}_{n}-\mathbf{R}_{m}\right|$, we take van der Waals interactions between two Rydberg atoms in states $a, b$ as $V_{a b}(d)=$ $C_{6}^{(a b)} /\left[\left(1+\delta_{a b}\right) d^{6}\right]$, for simplicity, where $C_{6}^{(a b)}$ is the dispersion coefficient and $\delta_{a b}$ Kronecker's delta. Between ground state atoms we assume the usual contact interaction $V_{g g}(d)=g \delta(d)$, where $g=4 \pi \hbar^{2} a_{s} / M$ with atom-atom $s$ wave scattering length $a_{s}$. Finally, ground state and Rydberg electrons interact via Fermi pseudopotentials $V_{g, r / R}(d)=$ $V_{0}\left|\Psi_{\nu / \nu^{\prime}}(d)\right|^{2}$, with $V_{0}=2 \pi \hbar^{2} a_{e} / m_{e}$ [29], electron mass $m_{e}$, electron-atom scattering length $a_{e}$ [30], and Rydberg orbital wave function $\Psi_{\nu / \nu^{\prime}}(d)$. Our examples will be based on Rb with $a_{e}=-0.849 \mathrm{~nm}$, assuming the Rydberg states $|r\rangle=|55 S\rangle$ and $|R\rangle=|76 S\rangle[31]$.

Now consider a scenario where $N_{\text {imp }}$ of the $N$ atoms have been excited to the impurity Rydberg state $|R\rangle$, denoting their locations by $\left\{\mathbf{x}_{n}\right\} \subset\left\{\mathbf{R}_{n}\right\}$. Many different random or deterministic location patterns can be created, depending on the method of excitation. We do not consider the excitation step, but refer to the literature on selective optical access [32-34], the exploitation of blockade effects [28,35-43], or condensate density dependent energy shifts $[16,23]$. Interactions $V_{R R}$ between two Rydberg impurities are important in the stage of impurity placement but can subsequently be neglected in the examples discussed here. The light-atom coupling in $\hat{H}_{\text {dress }}$ will cause long-range interactions for all atoms, which would otherwise be present only among Rydberg excited atoms $(|r\rangle,|R\rangle)$. Assuming far off-resonant coupling between $|g\rangle$ and $|r\rangle$, so that $|\alpha| \ll 1$ for $\alpha=\Omega(t) /(2 \Delta)$, we determine these interactions using fourth order perturbation theory in $\Omega(t)$. Calculating the energy shift $\Delta E_{\mathbf{g R}}$ of the state $|0\rangle=|\mathbf{g R}\rangle$ where the $N_{\text {imp }}$ atoms at locations $\mathbf{x}_{n}$ are in $|R\rangle$ and the other $\bar{N}=N-N_{\text {imp }}$ atoms in $|g\rangle$, we obtain $\Delta E_{\mathbf{g R}}=\alpha^{2} E_{(2)}+\alpha^{4} E_{(4)}$, where $E_{(2)}=\Delta \sum_{n}^{\bar{N}}\left(1-\sum_{m}^{N_{\text {imp }}} V_{r R}\left(\left|\mathbf{R}_{n}-\mathbf{x}_{m}\right|\right) / \Delta\right)^{-1}$. We find $E_{(4)}$, in comparison, negligible; see Ref. [44].

In this Letter, impurities will only affect dynamics for very short times, such that their motion can be assumed frozen in space [45], and they also do not undergo state changes. Similar to Ref. [6], we merge the effective interactions obtained through the laser dressing with the direct interactions between atoms contained in Eq. (1) $\left(V_{g R}, V_{g g}\right)$ to arrive at the following effective GrossPitaevskii equation (GPE) for the dressed and condensed ground state atoms in the presence of Rydberg impurities:

$$
\begin{aligned}
i \hbar \frac{\partial}{\partial t} \phi(\mathbf{R})= & \left(-\frac{\hbar^{2}}{2 m} \nabla^{2}+W(\mathbf{R})+g|\phi(\mathbf{R})|^{2}\right. \\
& +\left[U_{\text {eff }}^{(2)}\left(\mathbf{R},\left\{\mathbf{x}_{m}\right\}, t\right)\right. \\
& \left.\left.+\sum_{m}^{N_{\text {imp }}} V_{0}\left|\Psi\left(\left|\mathbf{R}-\mathbf{x}_{m}\right|\right)\right|^{2}\right]\right) \phi(\mathbf{R}), \\
U_{\text {eff }}^{(2)}= & \alpha^{2}(t) \Delta\left[1-\sum_{m}^{N_{\text {imp }}} V_{r R}\left(\left|\mathbf{R}-\mathbf{x}_{m}\right|\right) / \Delta\right]^{-1} .
\end{aligned}
$$

Here, the presence of a few impurity atoms that are fully in a Rydberg state causes strong, long-range interactions with the remaining atoms, which can be treated as external single body potential for the condensate. The corresponding terms in Eq. (2) are the first in square brackets and will be the central tool of the present Letter. Note that the dominant part $E_{(2)}$ is orders of magnitude larger than the dressed interaction between condensate atoms, causing quite different physics than the latter [6]. The induced potentials are sketched in Fig. 1(c) as a red line $\left(U_{\text {eff }}^{(2)}\right.$, using signs $\left.V_{r R} / \Delta<0\right)$ and a blue line $\left[|\Psi(|\mathbf{R}|)|^{2}\right]$ for a single impurity. Either potential is associated with an important length scale. The plateau of the dressing induced potential extends to the critical radius $r_{c}=\left|C_{6}^{(r R)} / \Delta\right|^{1 / 6}$, which also sets the width of the region of the potential drop. The extent of the direct interaction potential $V_{g R}$ is the radius of the Rydberg electron orbital $\Psi_{\nu^{\prime}}$ of the impurity, $r_{0} \approx a_{0} \nu^{\prime 2}$, with $a_{0}$ being the Bohr radius. We focus on parameters for which molecular resonances are avoided and also $r_{c}>r_{0}$ 
[46-48]. Although included in our solutions of Eq. (2) [49], the direct interactions $V_{g R}$ then play a minor role. It has been shown that many-body perturbative calculations as used here are valid only as long as there would be much less than one Rydberg excitation per blockade sphere $\left(N_{\mathrm{bl}} \ll 1\right)$ $[8,50]$, which will be satisfied here.

Our applications of Eq. (2) to phase-imprinting involve two dynamical stages: In a first short stage of duration $\tau_{\text {imp }} \sim 10 \mu \mathrm{s}$, the condensate order parameter $\phi$ acquires a dynamical phase $\varphi(\mathbf{R})$, such that $\phi(\mathbf{R}) \rightarrow \exp [i \varphi(\mathbf{R})] \phi(\mathbf{R})$, with $\varphi(\mathbf{R})=-U_{\text {eff }}^{(2)}\left(\mathbf{R},\left\{\mathbf{x}_{m}\right\}\right) \tau_{\text {imp. }}$. The time $\tau_{\text {imp }}$ and strength of $U_{\text {eff }}{ }^{(2)}$ are such that other energies can be neglected. Only in this stage are dressed interactions enabled through $\hat{H}_{\text {dress }}$. In a much longer second stage ( $t \sim 10 \mathrm{~ms}$ ), the condensate evolves according to the usual GPE, and the initially imprinted phase profile is typically transformed into condensate flow and/or density variations. We consider two examples that highlight the main strengths of Rydberg phase-imprinting: transferring entanglement from a Rydberg system onto a BEC, and inferring the geometry of a collection of Rydberg impurities in a cold gas.

Entanglement transfer.-We first consider a 1D arrangement of an ${ }^{85} \mathrm{Rb}$ BEC bright soliton (see Refs. [51,52] and the references therein) with $\bar{N}=400$ atoms, located between two individual atoms, which are each tightly trapped in their own optical tweezer at $x_{1,2}$, with position spread $\sigma_{\text {con }}=0.05 \mu \mathrm{m}$. The atoms outside the condensate are referred to as control atoms. As shown in Fig. 2, the

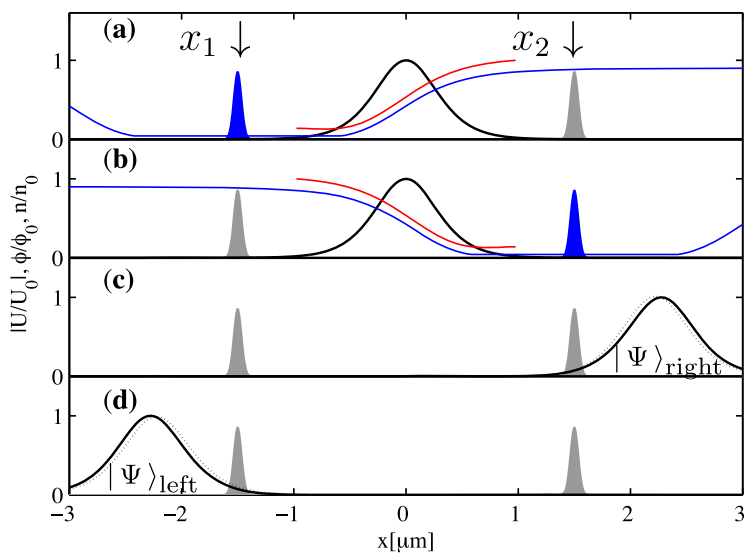

FIG. 2 (color online). Entanglement transfer from two control Rydberg atoms onto a mesoscopic BEC cloud. The position space density of control atoms is shown as shaded curves: blue for state $|R\rangle$ and gray for state $|g\rangle$. The condensate density for a soliton is shown as a thick black line. (Blue) Dressing potential $\left|U_{\mathrm{eff}}^{(2)}\right|$. (Red) Condensate phase $\varphi$ after imprinting. We show all quantities with an arbitrary normalization to fit the same axis. (a),(b) Initial state at $t=\tau_{\text {imp }}, \quad(\mathrm{c}),(\mathrm{d})$ final state at $t=\tau_{\text {imp }}+\tau_{\text {mov }}$. (a),(c) show the control atom configuration $|R g\rangle$, (b),(d) show $|g R\rangle$. For each configuration, we model condensate evolution separately using Eq. (2) [55]. The dotted line in (c),(d) is for shifted control atom positions $x_{i}+2 \sigma_{\text {con }}$. control atoms are separated by a distance $D=\left|x_{1}-x_{2}\right|=$ $3 \mu \mathrm{m}$. The soliton requires attractive contact interactions, $g<0$, which can be achieved using the Feshbach resonance [53] at $B \sim 155 \mathrm{G}$ in ${ }^{85} \mathrm{Rb}$ [54]; we assume $a_{s}(B)=$ $-5.33 \mathrm{~nm}$ [55]. Only the control atoms are now driven into the Rydberg state $|R\rangle=\left|\nu^{\prime}\right\rangle$ under blockade conditions, resulting in an entangled two-body state $|+\rangle=$ $(|g R\rangle+|R g\rangle) / \sqrt{2}$. Subsequently, we enable the dressing coupling $\Omega(t)$ to the state $|r\rangle$ for the bulk soliton, resulting in the dressed potential sketched blue in Figs. 2(a) and 2(b), which depends on whether the left or right control atom was originally excited. After an adiabatically enabled and disabled imprinting period $\tau_{\text {imp }}=36 \mu$ s, with $\Omega(t) / h=$ $3 \mathrm{MHz}$ and $\Delta / h=-500 \mathrm{MHz}$, the condensate has acquired the phase profile shown in red. Following deexcitation of the control atoms, we allow $\tau_{\mathrm{mov}}=2 \mathrm{~ms}$ of free evolution according to the first line of Eq. (2), i.e., $\Omega(t)=0$. After $\tau_{\text {mov }}$, the soliton has moved by about $2 \mu \mathrm{m}$ to the left or right, depending on the imprinted phase profile, as shown in Figs. 2(c) and 2(d). Let us denote the many-body wave function of the gas for these two cases as $|\Psi\rangle_{\text {left/right }}$. The entire process should be quantum coherent, since the initial imprinting happens well before a control Rydberg state or a dressed Rydberg state from the condensate would decay [56], resulting in a final many-body state $\left|\Psi_{+}\right\rangle=\left(|\Psi\rangle_{\text {left }}+|\Psi\rangle_{\text {right }}\right) / \sqrt{2}$.

The tightly trapped control atoms [58] and the small length scales [32] are technical challenges for the above proposal. However, when these are overcome, one obtains a mesoscopic entangled state [22,59-64], where the entire soliton of $\bar{N}$ atoms is in a superposition of two different locations, as proposed in Refs. [22,65].

The superposition nature of the resulting state can be proven interferometrically $[22,65,66]$ upon recombination, for which one would additionally place the soliton into a weak harmonic trap $W(\mathbf{R})$. Decoherence processes during the creation of such a highly entangled many-body state limit $\bar{N}$, but they are small for our choice here [67]. A full quantum many-body treatment including coherence between control atom states and condensate atoms may be a subject of further research.

Rydberg crystal imprinting.-The maximally entangled state $\left|\varphi_{+}\right\rangle$is but one example of entanglement arising due to strong Rydberg-Rydberg interactions. Another example is given by spatially ordered (crystal) structures formed by large numbers of Rydberg excitations in a cold gas $[28,40,42,68-70]$. We show now that Rydberg phaseimprinting in the presence of such structures leads to condensate momentum spectra that allow the reconstruction of the locations of impurities.

Let us consider a 2D model [71] of a BEC confined in a pancake shaped harmonic trap $W(\mathbf{R})=m\left[\omega_{r}^{2}\left(x^{2}+y^{2}\right)+\right.$ $\left.\omega_{z}^{2} z^{2}\right] / 2$, with frequencies $\omega_{z} \gg \omega_{r}$ and $a_{s}=5.5 \mathrm{~nm}$; thus, $g>0$. Using a scheme as discussed in Ref. [28], $N_{\text {imp }}$ 
impurities can be arranged, for example, in a crystal-like structure within the condensate cloud.

For a distribution of impurities as shown in Fig. 3(a), we numerically solve Eq. (2) [72,73] enabling the potentials $U_{\text {eff }}{ }^{(2)}$ and $V_{g R}$ [49] for a short imprinting period $\tau_{\text {imp }}=$ $18.5 \mu \mathrm{s}$ only, using $\Omega / h=4 \mathrm{MHz}, \Delta / h=-150 \mathrm{MHz}$. This is followed by evolution under the influence of the contact interactions, but with a disabled harmonic trap and the impurities assumed removed via field ionization $[16,74]$. At some final time where momentum spectra no longer significantly change, we plot the expected time-offlight images in Fig. 3. We also show the position space density shortly after phase-imprinting.

If the effect of atomic collisions, described by the nonlinear term $g|\phi(\mathbf{R})|^{2}$, is not too large, the final momentum spectrum is roughly the Fourier transform of $\phi(\mathbf{R})=\sqrt{n_{\text {ini }}(\mathbf{R})} \exp [i \varphi(\mathbf{R})]$, where $n_{\text {ini }}(\mathbf{R})$ is the known initial atom density in the trap, and $\varphi(\mathbf{R})$ the phase profile generated through imprinting and shown in Fig. 3(b). A standard phase-retrieval algorithm $[75,76]$ is then able to recover the phase profile as shown in Fig. 3(e), from which impurity positions can clearly be inferred. The algorithm relies on iterative Fourier transforms involving two known quantities: the final time-of-flight image from which the modulus of the condensate order parameter is extracted $|\tilde{\phi}(k)|$, and the initial condensate density $n_{\text {ini }}(\mathbf{R})$. We briefly describe the algorithm in Ref. [44].

We find that simple phase retrieval fails for larger condensate densities than in Fig. 3 (and other parameters

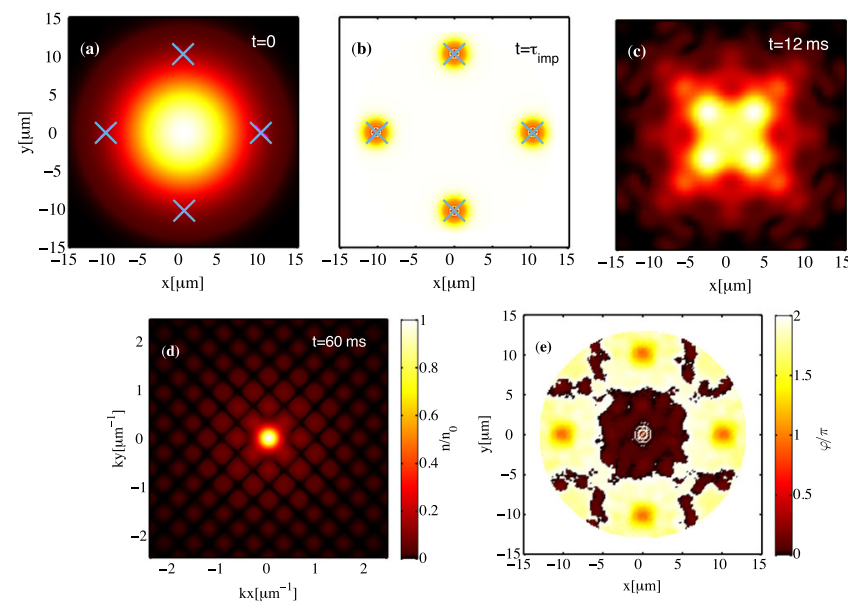

FIG. 3 (color online). Determining the spatial arrangement of Rydberg impurities (shown as blue crosses) via phase-imprinting in a $2 \mathrm{D}{ }^{87} \mathrm{Rb} \mathrm{BEC}$ of $N=100$ atoms in a trap with $\omega_{r}=$ $(2 \pi) 2 \mathrm{~Hz}$ and $\omega_{z}=(2 \pi) 100 \mathrm{~Hz}$. Color bar for (a),(c),(d) [see (d)], where $n_{0}$ is the respective peak density, color bar for (b),(e) [see (e)]. (a) Initial condensate density $\rho=|\phi(\mathbf{R})|^{2}$. (b) Condensate phase $\varphi$ following phase-imprinting $\left(t=\tau_{\text {imp }}\right)$. (c) Condensate density shortly after imprinting $\left(t=\tau_{\text {imp }}+12 \mathrm{~ms}\right)$.

(d) Final momentum spectrum $|\tilde{\phi}(k)|(t=60 \mathrm{~ms})$. (e) Phase profile reconstructed from (d) as described in the text. the same) due to condensate self-interactions. This might be remedied by more sophisticated variants of the phaseretrieval algorithm [76], or modifications of atomic interactions using a Feshbach resonance [53]. One could then use larger atom clouds, in which case impurity locations can be recovered from a single image, as in Fig. 3(d), without the need for image alignment in an ensemble average, as in Ref. [42].

The setup just discussed complements Rydberg crystal detection based on single-atom addressing [42] or electromagnetically induced transparency [24-26] by working with a bulk gas and moving the signal from the light to the atomic density. Beyond crystal detection, it enables phase profiles that are otherwise difficult to achieve, for example those akin to Fig. 3(b) arising from a crystalline impurity distribution on the surface of a 3D sphere.

Conclusions and outlook.-We proposed a novel phaseimprinting technique for Bose-Einstein condensates, employing long-range interactions between condensate atoms and embedded Rydberg excited impurity atoms, created by coupling condensate atoms far off-resonantly to another Rydberg state. The scheme offers functionalities beyond existing imprinting methods, as it allows mapping of entanglement from few-body Rydberg states onto the whole atom cloud and strengthens BEC as a diagnostic tool for detecting Rydberg excitations in an ensemble of atoms. We illustrate the former through a proposal for the creation of a mesoscopic entangled state in the position of a cloud of atoms and the latter by exploring the link between Rydberg crystal structures in a condensate, and momentum space spectra after phase-imprinting.

Combing the techniques discussed here with imprinting effects by a Rydberg electron in a larger orbital may offer additional possibilities due to the unusual shape of the Rydberg orbital [16-18]. Other interesting physics might arise from the interplay of phase-imprinting and controlled impurity motion [77-81].

We gladly acknowledge our fruitful discussions with Igor Lesanovsky, Thomas Pohl, Rick van Bijnen, and Shannon Whitlock, as well as the EU financial support received from the Marie Curie Initial Training Network (ITN) "COHERENCE." W. L. is supported through the Nottingham Research Fellowship by the University of Nottingham.

[1] Ł. Dobrek, M. Gajda, M. Lewenstein, K. Sengstock, G. Birkl, and W. Ertmer, Phys. Rev. A 60, R3381 (1999).

[2] J. Denschlag, J. E. Simsarian, D. L. Feder, C. W. Clark, L. A. Collins, J. Cubizolles, L. Deng, E. W. Hagley, K. Helmerson, W. P. Reinhardt et al., Science 287, 97 (2000).

[3] S. Burger, K. Bongs, S. Dettmer, W. Ertmer, K. Sengstock, A. Sanpera, G. V. Shlyapnikov, and M. Lewenstein, Phys. Rev. Lett. 83, 5198 (1999).

[4] J. Ruostekoski, Phys. Rev. A 61, 041603 (2000). 
[5] L. Santos, G. V. Shlyapnikov, P. Zoller, and M. Lewenstein, Phys. Rev. Lett. 85, 1791 (2000).

[6] N. Henkel, R. Nath, and T. Pohl, Phys. Rev. Lett. 104, 195302 (2010).

[7] G. Pupillo, A. Micheli, M. Boninsegni, I. Lesanovsky, and P. Zoller, Phys. Rev. Lett. 104, 223002 (2010).

[8] J. Honer, H. Weimer, T. Pfau, and H. P. Büchler, Phys. Rev. Lett. 105, 160404 (2010).

[9] F. Maucher, N. Henkel, M. Saffman, W. Królikowski, S. Skupin, and T. Pohl, Phys. Rev. Lett. 106, 170401 (2011).

[10] S. Wüster, C. Ates, A. Eisfeld, and J. M. Rost, New J. Phys. 13, 073044 (2011).

[11] J. E. Johnson and S. L. Rolston, Phys. Rev. A 82, 033412 (2010).

[12] J. B. Balewski, A. T. Krupp, A. Gaj, S. Hofferberth, R. Löw, and T. Pfau, New J. Phys. 16, 063012 (2014).

[13] Y.-Y. Jau, A. M. Hankin, T. Keating, I. H. Deutsch, and G. W. Biedermann, arXiv:1501.03862.

[14] R. Heidemann, U. Raitzsch, V. Bendkowsky, B. Butscher, R. Löw, and T. Pfau, Phys. Rev. Lett. 100, 033601 (2008).

[15] M. Viteau, M. G. Bason, J. Radogostowicz, N. Malossi, D. Ciampini, O. Morsch, and E. Arimondo, Phys. Rev. Lett. 107, 060402 (2011).

[16] J. B. Balewski, A. T. Krupp, A. Gaj, D. Peter, H. P. Büchler, R. Löw, S. Hofferberth, and T. Pfau, Nature (London) 502, 664 (2013).

[17] A. Gaj, A. T. Krupp, J. B. Balewski, R. Löw, S. Hofferberth, and T. Pfau, Nat. Commun. 5, 4546 (2014).

[18] T. Karpiuk, M. Brewczyk, K. Rzążewski, J. B. Balewski, A. T. Krupp, A. Gaj, R. Löw, S. Hofferberth, and T. Pfau, New J. Phys. 17, 053046 (2015).

[19] D. Jaksch, J. I. Cirac, P. Zoller, S. L. Rolston, R. Côté, and M. D. Lukin, Phys. Rev. Lett. 85, 2208 (2000).

[20] R. Mukherjee, J. Millen, R. Nath, M. P. A. Jones, and T. Pohl, J. Phys. B 44, 184010 (2011).

[21] S. Wüster, S. Möbius, M. Genkin, A. Eisfeld, and J.-M. Rost, Phys. Rev. A 88, 063644 (2013).

[22] C. Weiss and Y. Castin, Phys. Rev. Lett. 102, 010403 (2009).

[23] S. Middelkamp, I. Lesanovsky, and P. Schmelcher, Phys. Rev. A 76, 022507 (2007).

[24] B. Olmos, W. Li, S. Hofferberth, and I. Lesanovsky, Phys. Rev. A 84, 041607(R) (2011).

[25] G. Günter, M. Robert-de-Saint-Vincent, H. Schempp, C. S. Hofmann, S. Whitlock, and M. Weidemüller, Phys. Rev. Lett. 108, 013002 (2012).

[26] G. Günter, H. Schempp, M. Robert-de-Saint-Vincent, V. Gavryusev, S. Helmrich, C. S. Hofmann, S. Whitlock, and M. Weidemüller, Science 342, 954 (2013).

[27] J. Wang, M. Gacesa, and R. Côté, Phys. Rev. Lett. 114, 243003 (2015).

[28] T. Pohl, E. Demler, and M. D. Lukin, Phys. Rev. Lett. 104, 043002 (2010).

[29] C. H. Greene, A. S. Dickinson, and H. R. Sadeghpour, Phys. Rev. Lett. 85, 2458 (2000).

[30] The momentum dependence of which we neglect here.

[31] For these states we find $C_{6}^{(r r)}=43.4 \mathrm{GHz} \times \mu \mathrm{m}^{6}$, $C_{6}^{(r R)}=6.5 \mathrm{GHz} \times \mu \mathrm{m}^{6}, C_{6}^{(R R)}=1880.6 \mathrm{GHz} \times \mu \mathrm{m}^{6}$.

[32] F. Nogrette, H. Labuhn, S. Ravets, D. Barredo, L. Béguin, A. Vernier, T. Lahaye, and A. Browaeys, Phys. Rev. X 4, 021034 (2014).
[33] M. Schlosser, S. Tichelmann, J. Kruse, and G. Birkl, Quantum Inf. Process. 10, 907 (2011).

[34] R. M. W. van Bijnen, C. Ravensbergen, D. J. Bakker, G. J. Dijk, S. J. J. M. F. Kokkelmans, and E. J. D. Vredenbregt, New J. Phys. 17, 023045 (2015).

[35] M. Gärttner, K. P. Heeg, T. Gasenzer, and J. Evers, Phys. Rev. A 88, 043410 (2013).

[36] H. Weimer, R. Löw, T. Pfau, and H. P. Büchler, Phys. Rev. Lett. 101, 250601 (2008).

[37] S. Wüster, J. Stanojevic, C. Ates, T. Pohl, P. Deuar, J. F. Corney, and J. M. Rost, Phys. Rev. A 81, 023406 (2010).

[38] I. Lesanovsky and J. P. Garrahan, Phys. Rev. A 90, 011603 (2014).

[39] S. Bettelli, D. Maxwell, T. Fernholz, C. S. Adams, I. Lesanovsky, and C. Ates, Phys. Rev. A 88, 043436 (2013).

[40] R. M. W. van Bijnen, S. Smit, K. A. H. van Leeuwen, E. J. D. Vredenbregt, and S. J. J. M. F. Kokkelmans, J. Phys. B 44, 184008 (2011).

[41] H. Schempp, G. Günter, M. Robert-de-Saint-Vincent, C. S. Hofmann, D. Breyel, A. Komnik, D. W. Schönleber, M. Gärttner, J. Evers, S. Whitlock et al., Phys. Rev. Lett. 112, 013002 (2014).

[42] P. Schauß, M. Cheneau, M. Endres, T. Fukuhara, S. Hild, A. Omran, T. Pohl, C. Gross, S. Kuhr, and I. Bloch, Nature (London) 491, 87 (2012).

[43] P. Schauß, J. Zeiher, T. Fukuhara, S. Hild, M. Cheneau, T. Macrí, T. Pohl, I. Bloch, and C. Gross, Science 347, 1455 (2015).

[44] See Supplemental Material at http://link.aps.org/ supplemental/10.1103/PhysRevLett.115.040401 for details on the derivation of dressed potentials and phase recovery.

[45] W. R. Anderson, J. R. Veale, and T. F. Gallagher, Phys. Rev. Lett. 80, 249 (1998).

[46] V. Bendkowsky, B. Butscher, J. Nipper, J. P. Shaffer, R. Löw, and T. Pfau, Nature (London) 458, 1005 (2009).

[47] V. Bendkowsky, B. Butscher, J. Nipper, J. B. Balewski, J. P. Shaffer, R. Löw, T. Pfau, W. Li, J. Stanojevic, T. Pohl et al., Phys. Rev. Lett. 105, 163201 (2010).

[48] W. Li, T. Pohl, J. Rost, S. T. Rittenhouse, H. R. Sadeghpour, J. Nipper, B. Butscher, J. B. Balewski, V. Bendkowsky, R. Löw et al., Science 334, 1110 (2011).

[49] To keep the numerics tractable, we include $V_{g, R}(r)$ only approximately. (i) Its amplitude, substantially exceeding other energy scales, is cut off at $\left|V_{g, R}(r)\right|=5 \Delta \alpha^{2}$. (ii) Its spatial profile is undersampled since numerical discretizations of space that can represent the time-of-flight expansion required for Fig. 3 cannot simultaneously resolve the very fine oscillations of the Rydberg wave function. We have separately confirmed that the relative strength of the amplitude and phase perturbations of the condensate wave function after the imprinting period are representative.

[50] In a homogeneous 3D system we have $N_{\mathrm{bl}}=\alpha^{2} n_{0} V_{\mathrm{bl}}$ atoms per blockade sphere $V_{\mathrm{bl}}=4 \pi r_{\mathrm{bl}}^{3} / 3$ (the volume in which one Rydberg excitation blocks another), where $n_{0}$ is the background atom density and $r_{b l}=\left|C_{6}^{(r r)} / \Delta\right|^{1 / 6}$ the blockade radius.

[51] Y.S. Kivshar and G. P. Agrawal, Optical Solitons: From Fibers to Photonic Crystals (Academic, San Diego, 2003). 
[52] B. J. Dąbrowska-Wüster, S. Wüster, and M. J. Davis, New J. Phys. 11, 053017 (2009).

[53] E. Timmermans, P. Tommasini, M. Hussein, and A. Kerman, Phys. Rep. 315, 199 (1999).

[54] E. A. Donley, N. R. Claussen, S. L. Cornish, J. L. Roberts, E. A. Cornell, and C. E. Wieman, Nature (London) 412, 295 (2001).

[55] For an effective 1D treatment, we replace $g \rightarrow g_{1 D}=$ $g\left(2 \pi \sigma_{\perp}^{2}\right)^{-1}, \sigma_{\perp}=\sqrt{\hbar / m / \omega_{\perp}}, \omega_{\perp}=(2 \pi) 200 \mathrm{~Hz}$.

[56] The lifetime of $|R\rangle$ is $\tau_{\nu^{\prime}=76} \approx 180 \mu$ s [57] and the effective dressed condensate lifetime is $\tau_{\text {eff }}=\tau_{\nu=55} /\left(\bar{N} \alpha^{2}\right) \approx 23 \mathrm{~ms}$.

[57] I. I. Beterov, I. I. Ryabtsev, D. B. Tretyakov, and V. M. Entin, Phys. Rev. A 79, 052504 (2009).

[58] L. Li, Y. O. Dudin, and A. Kuzmich, Nature (London) 498, 466 (2013).

[59] D. W. Hallwood, T. Ernst, and J. Brand, Phys. Rev. A 82, 063623 (2010).

[60] D. Gordon and C. M. Savage, Phys. Rev. A 59, 4623 (1999).

[61] J. A. Dunningham, K. Burnett, R. Roth, and W. D. Phillips, New J. Phys. 8, 182 (2006).

[62] S. Möbius, M. Genkin, A. Eisfeld, S. Wüster, and J. M. Rost, Phys. Rev. A 87, 051602(R) (2013).

[63] J. I. Cirac, M. Lewenstein, K. Mølmer, and P. Zoller, Phys. Rev. A 57, 1208 (1998).

[64] H. T. Ng, Phys. Rev. A 77, 033617 (2008).

[65] S. Möbius, M. Genkin, A. Eisfeld, S. Wüster, and J.-M. Rost, Phys. Rev. A 87, 051602(R) (2013).

[66] B. Gertjerenken, T. P. Billam, L. Khaykovich, and C. Weiss, Phys. Rev. A 86, 033608 (2012).
[67] For example, we expect a mean loss of 0.8 atoms from the soliton during $\tau_{\text {mov }}$, dominated by three-body recombination.

[68] H. Weimer, R. Löw, T. Pfau, and H. P. Büchler, Phys. Rev. Lett. 101, 250601 (2008).

[69] H. Weimer, R. Löw, T. Pfau, and H. P. Büchler, New J. Phys. 12, 103044 (2010).

[70] M. Gärttner, K. P. Heeg, T. Gasenzer, and J. Evers, Phys. Rev. A 88, 043410 (2013).

[71] The 2D interaction is $g \rightarrow g_{2 \mathrm{D}}=g / \sqrt{2 \pi \sigma_{\mathrm{z}}^{2}}$, where $\sigma_{\mathrm{z}}=\sqrt{\hbar /\left(m \omega_{z}\right)}$.

[72] G. R. Dennis, J. J. Hope, and M. T. Johnsson, Comput. Phys. Commun. 184, 201 (2013).

[73] G. R. Dennis, J. J. Hope, and M. T. Johnsson, http://www .xmds.org/ (2012).

[74] T.F. Gallagher, Rydberg Atoms (Cambridge University Press, Cambridge, England, 1994).

[75] R. W. Gerchberg and W. O. Saxton, Optik (Stuttgart) 35, 237 (1972).

[76] J. R. Fienup, Appl. Opt. 21, 2758 (1982).

[77] C. Ates, A. Eisfeld, and J. M. Rost, New J. Phys. 10, 045030 (2008).

[78] S. Möbius, S. Wüster, C. Ates, A. Eisfeld, and J. M. Rost, J. Phys. B 44, 184011 (2011).

[79] S. Wüster, C. Ates, A. Eisfeld, and J. M. Rost, Phys. Rev. Lett. 105, 053004 (2010).

[80] S. Wüster, A. Eisfeld, and J. M. Rost, Phys. Rev. Lett. 106, 153002 (2011).

[81] K Leonhardt and S Wüster and J.-M. Rost, Phys. Rev. Lett. 113, 223001 (2014). 